\title{
Near-infrared fundus autofluorescence-visualized melanin in the choroidal abnormalities of neurofibromatosis type I
}

This article was published in the following Dove Press journal:

Clinical Ophthalmology

26 July 2012

Number of times this article has been viewed

\section{Tomoko Ueda-Consolvo \\ Akio Miyakoshi \\ Hironori Ozaki \\ Satoshi Houki \\ Atsushi Hayashi}

Department of Ophthalmology,

Graduate School of Medicine and Pharmaceutical Sciences, University

of Toyama, Toyama, Japan
Correspondence: Atsushi Hayashi Department of Ophthalmology, Graduate School of Medicine and Pharmaceutical Sciences, University of Toyama, 2630 Sugitani, Toyama, 930-0194, Japan

Tel +8I 764347363

Fax+8I 764345037

Email ahayashi@med.u-toyama.ac.jp
Purpose: To report a series of three cases of neurofibromatosis type 1 examined by near-infrared fundus autofluorescence (NIR-AF) with a scanning laser ophthalmoscope and spectral-domain optical coherence tomography (OCT) to show the characteristics of choroidal abnormalities.

Methods: Retrospective case series. Six eyes of three patients were examined by conventional fundus examinations, near-infrared monochromatic light reflectance (NIR-R) and NIR-AF, OCT, fluorescein angiography, and indocyanine green angiography.

Results: All eyes showed multiple bright patchy regions in the choroid of the posterior pole with NIR-R. NIR-AF revealed high fluorescent regions of similar sizes at fundus locations identical to those shown by NIR-R. In one case, hypofluorescent regions were shown by indocyanine green angiography in the bright fluorescent region shown by NIR-AF. The other two cases showed no abnormality under conventional fundus examination or fluorescein angiography. OCT images crossing the bright patchy region showed irregular hyper-reflectivity in the choroid in two cases and hyporeflectivity in one case.

Conclusions: NIR-AF demonstrated that dense melanin was included in the choroidal nodules of neurofibromatosis type 1 . The choroidal nodules showed hyper- or hyporeflectivity in the choroid on OCT, which did not affect the retinal structure.

Keywords: near-infrared fundus autofluorescence, neurofibromatosis type 1, choroidal nodule, melanin

\section{Introduction}

Neurofibromatosis type 1 (NF1) is an autosomal dominant disorder with a high mutation rate. The most common ocular feature of NF1 is the Lisch nodules of the iris, which are described as "iris hamartomas." Other ocular manifestations include optic gliomas, orbital neurofibromas, café-au-lait spots on the eyelids, congenital absence of the sphenoid wing, and congenital glaucoma. ${ }^{1}$ The choroidal abnormalities in NF1 had been thought to be rare, but bright multiple patchy regions were observed in all of the eyes examined by near-infrared monochromatic light reflectance (NIR-R) with a scanning laser ophthalmoscope. ${ }^{2}$ Recently, the cutoff value of choroidal nodules detected by NIR-R was reported to be 1.5 choroidal nodules. ${ }^{3}$ Thus, NIR-R has been required to detect choroidal nodules in NF1 patients, and the choroid is one of the structures most commonly affected by NF1. ${ }^{2-4}$

The source of near-infrared fundus autofluorescence (NIR-AF) was shown to be the melanosomes in the retinal pigment epithelium (RPE) and the choroid. ${ }^{5}$ Other studies demonstrated that NIR-AF visualized the melanin in age-related macular degeneration and other retinal diseases. ${ }^{6,7}$ 
In this study we examined the usefulness of NIR-AF for detecting melanin in the choroidal nodules of NF1 patients and the correlation between choroidal nodules and the appearance of spectral-domain optical coherence tomography (OCT).

\section{Patients and methods}

We studied six eyes of three patients with NF1 (three women). These consecutive patients visited Toyama University Hospital, Toyama, Japan, between March 2011 and December 2011. The diagnosis of NF1 was based on stringent National Institutes of Health criteria. All patients underwent comprehensive ophthalmologic examinations, including measurement of the best-corrected decimal visual acuity and intraocular pressure, slit-lamp biomicroscopy, fundus color photography, and OCT (RS-3000 Advance, NIDEK Co, Ltd, Aichi, Japan, and Cirrus ${ }^{\mathrm{TM}}$ HD-OCT, Carl Zeiss Meditec Inc, Dublin, CA). We also performed fluorescein angiography (FA) and indocyanine green angiography (ICGA). Fundus examinations of NIR-R and NIR-AF were performed in all patients using a confocal scanning laser ophthalmoscope (F-10, NIDEK Co, Ltd). NIR-AF was obtained with an excitation filter of $790 \mathrm{~nm}$ and a barrier filter covering $820 \mathrm{~nm}$ to $880 \mathrm{~nm}$, filters that were normally used for ICGA.

\section{Results}

The characteristics of the six eyes of three patients are listed in Table 1. The patients' ages ranged from 20 to 46 years old. The best-corrected decimal visual acuity of the six eyes ranged from 0.4 to 1.2. Although we searched causes for the decreased visual acuity by visual fields and brain magnetic resonance imaging, we could not identify a clear cause for the decreased visual acuity. Lisch nodules were noted in all eyes. Conventional fundus examinations and fundus color photography did not show any abnormalities in two young patients; however, the mother of case 1, who was
46 years old, showed a slight alteration of the RPE in the posterior pole.

Figure 1 shows images of the FA and ICGA of case 1. No abnormal regions were observed in the fundus with FA (Figure 1A and C), but there were several regions with a slight hypofluorescence by ICGA (arrows in Figure 1B and D). NIR-R revealed many bright patchy regions in the posterior pole of both eyes in case 1 (the right eye is shown in Figure 2A). NIR-AF demonstrated hyperfluorescent regions of similar sizes at fundus locations identical to those where the bright patchy regions were detected by NIR-R (Figure 2B), although there was no apparent abnormality in a color fundus photograph (Figure 2C). An OCT image crossing the bright patchy regions showed an irregular hyper-reflective region in the choroid, although there was no abnormality in the retinal structures, such as the inner segment and outer segment junction line (Figure 2D).

Case 2, who was the mother of case 1, showed hypofluorescent spots in ICGA and hyperfluorescence in the posterior pole due to RPE alterations in FA (arrows in Figure 3A and $\mathrm{B}$ ). In a color fundus photograph, color changes due to RPE alterations were observed (Figure 3C). In Figure 3D, NIR-R revealed multiple bright patchy regions, characteristic of NF1, in the posterior pole of the right eye of case 2. An OCT image showed an irregular hyporeflective region in the choroid crossing at the bright patchy region, and no abnormal changes were observed in the retinal structure (Figure $3 \mathrm{E}$ ). As in case 1, similar hyperfluorescent regions at fundus locations identical to those shown by NIR-R were observed with NIR-AF (Figure 3F).

Case 3 also revealed bright patchy regions in the posterior pole of the left eye with NIR-R (Figure 4A), and hyperfluorescent regions of similar sizes were detected with NIR-AF (Figure 4B). An OCT image showed an irregular hyperreflective region in the choroid crossing at the bright patchy region, but there was no abnormality in the retinal structure (Figure 4C). Figure 4D showed a color fundus photograph without apparent abnormality.

Table I Characteristics of three cases with neurofibromatosis type I

\begin{tabular}{|c|c|c|c|c|c|c|c|c|c|c|c|}
\hline \multirow[t]{2}{*}{ Case } & \multirow[t]{2}{*}{ Age } & \multirow[t]{2}{*}{ Sex } & \multirow[t]{2}{*}{ Eye } & \multirow[t]{2}{*}{ BCVA } & \multicolumn{6}{|c|}{ Choroidal abnormalities } & \multirow[t]{2}{*}{ Lisch nodule } \\
\hline & & & & & Color & FA & ICGA & NIR-AF & NIR-R & $\overline{\text { OCT }}$ & \\
\hline \multirow[t]{2}{*}{ I } & 20 & $\mathrm{~F}$ & $\mathrm{R}$ & 0.5 & - & - & + & + & + & Hyper & + \\
\hline & & & $\mathrm{L}$ & 0.4 & - & - & + & + & + & Hyper & + \\
\hline \multirow[t]{2}{*}{2} & 46 & $\mathrm{~F}$ & $\mathrm{R}$ & 1.2 & + & + & + & + & + & Нуро & + \\
\hline & & & $\mathrm{L}$ & 1.0 & + & + & + & + & + & Нуро & + \\
\hline \multirow[t]{2}{*}{3} & 28 & $\mathrm{~F}$ & $\mathrm{R}$ & 0.8 & - & - & - & + & + & Hyper & + \\
\hline & & & $\mathrm{L}$ & 1.0 & - & - & - & + & + & Hyper & + \\
\hline
\end{tabular}

Abbreviations: BCVA, best corrected decimal visual acuity; FA, fluorescein angiography; ICGA, indocyanine green angiography; NIR-AF, near-infrared fundus autofluorescence; NIR-R, near-infrared monochromatic light reflectance; OCT, optical coherence tomography; F, female; R, right; L, left. 

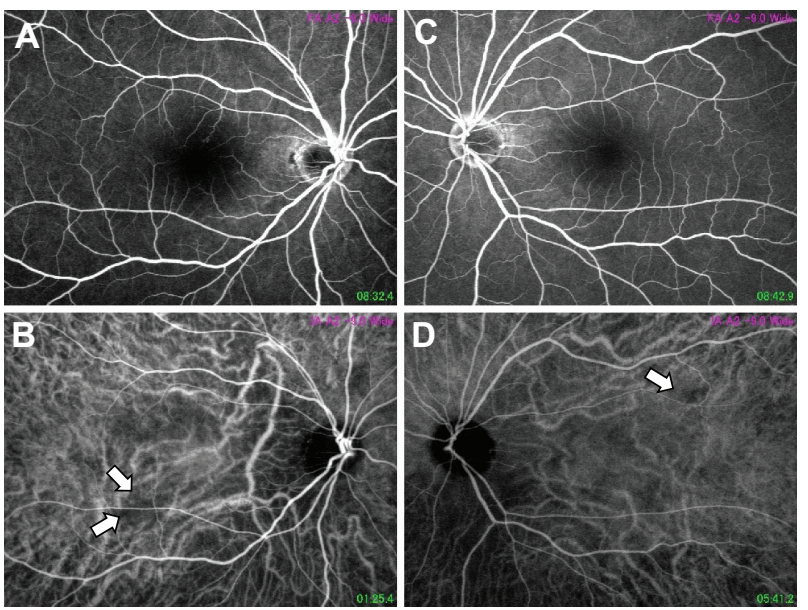

Figure I Fundus photographs of fluorescein angiography (A and $\mathbf{C}$ ) and indocyanine green angiography ( $\mathbf{B}$ and $\mathbf{D}$ ) of case I. Although no abnormality was observed in either eye by fluorescein angiography $(\mathbf{A})$ right eye, (C) left eye), several hypofluorescent regions were observed by indocyanine green angiography (arrows in (B and $\mathbf{D}$ ), (B): right eye, (D): left eye).

\section{Discussion}

Fundus examination with NIR-R using a confocal scanning laser ophthalmoscope was shown to effectively visualize choroidal nodules in all examined eyes of NF1 patients. ${ }^{2}$ In a recent study with a large number of NF1 patients, choroidal nodules were detected in $82 \%$ of NF1 patients with confocal NIR imaging, compared with $7 \%$ of healthy patients. ${ }^{3}$ These results suggest that a near-infrared fundus examination seems essential to visualizing choroidal abnormalities in NF1 patients. In this study we similarly showed multiple bright
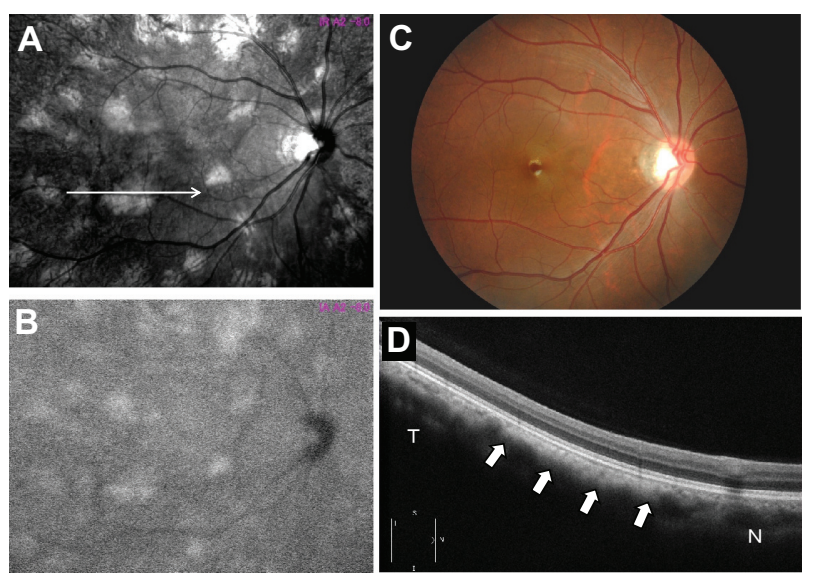

Figure 2 Fundus photographs of the right eye of case I by reflectance of a near-infrared monochromatic light resistance $(\mathbf{A})$, near-infrared fundus autofluorescence (B), color fundus photograph (C), and optical coherence tomography (D). Near-infrared fundus autofluorescence (B) shows similar hyperfluorescent regions at locations identical to those revealed by near-infrared monochromatic light resistance (A).

Note: An optical coherence tomography image crossing the bright patchy regions (arrow in Figure 2A) shows an irregular hyper-reflectance focus in the choroid (arrows in Figure 2D).

Abbreviations: $\mathrm{N}$, nasal; $\mathrm{T}$, temporal.
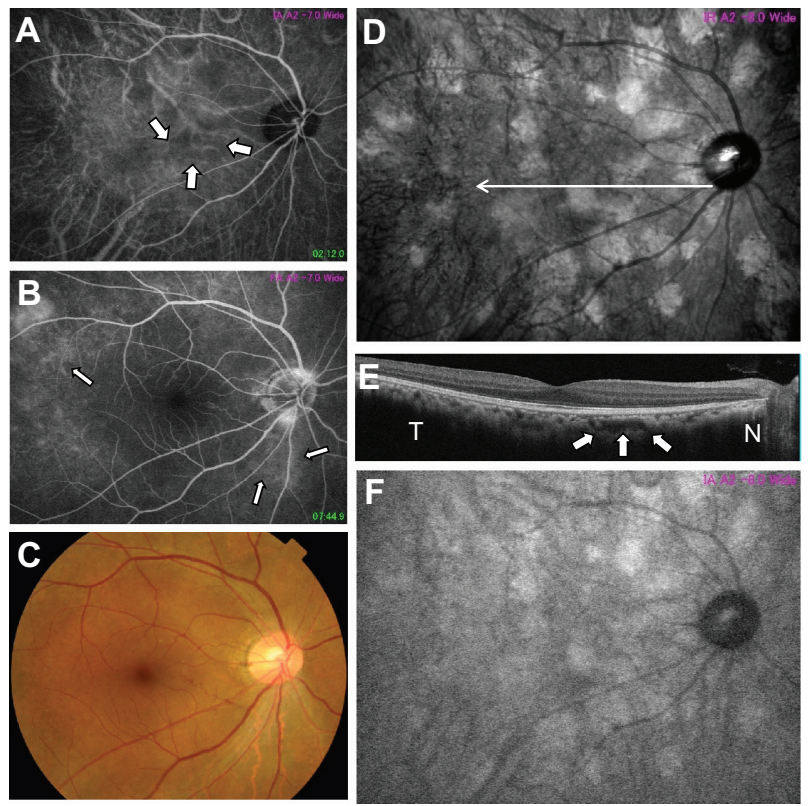

Figure 3 Fundus photographs of the right eye by indocyanine green angiography (A), fluorescein angiography (B), color fundus photograph (C), near-infrared monochromatic light resistance (D), optical coherence tomography image (E), and near-infrared fundus autofluorescence $(\mathbf{F})$ of case 2. Arrows in Figure 3A show hypofluorescent regions by indocyanine green angiography. Arrows in Figure 3B show hyperfluorescent regions by fluorescein angiography. An optical coherence tomography image crossing the bright patchy regions (arrow in Figure 3D) shows an irregular hyporeflectance focus in the choroid (arrows in Figure 3E). Near-infrared fundus autofluorescence $(\mathbf{F})$ shows hyperfluorescent regions of similar sizes at locations identical to those revealed by near-infrared monochromatic light resistance (D).

Abbreviations: $\mathrm{N}$, nasal; $\mathrm{T}$, temporal.

patchy regions in the fundus of the three patients using nearinfrared fundus examinations. In histopathological studies, ovoid bodies in the choroid consisted of hyperplastic Schwann cells, melanocytes, and ganglion cells in a neurofibromatosis patient. ${ }^{8,9}$ In vivo imaging of human skin via reflectance of a
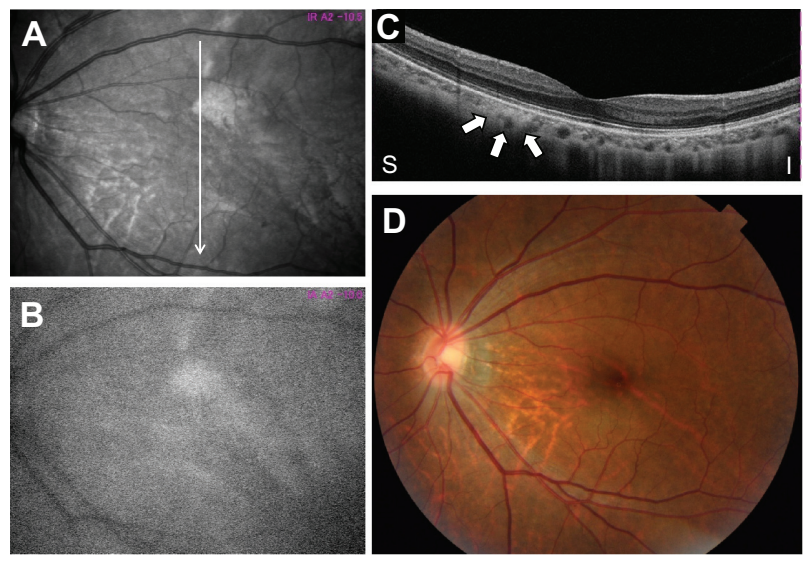

Figure 4 Fundus photographs of the left eye by near-infrared monochromatic light resistance (A), near-infrared fundus autofluorescence (B), optical coherence tomography (C), and color fundus photograph (D) of case 3. An optical coherence tomography image crossing the bright patchy regions (arrow in Figure 4A) shows an irregular hyper-reflectance focus in the choroid (Figure 4C).

Abbreviations: I, inferior; S, superior. 
near-infrared light with confocal scanning laser microscopy visualized blood cells in the deeper capillaries and heavily pigmented cells by increased backscattering. ${ }^{10}$ Therefore, the fundus appearance on NIR-R may reflect not only increased melanocytes but also choroidal vessels and blood cells. However, a brighter reflectance of confocal imaging of the skin was observed in darkly pigmented skin than in lightly pigmented skin, because melanin provides strong cytoplasmic contrast effects. ${ }^{10}$ As the source of NIR-AF was shown to be melanosomes in the RPE and choroids, ${ }^{5}$ multiple bright regions in the fundus revealed by NIR-AF reflected dense melanin in the choroidal nodules. ${ }^{6,7,11}$ Thus, NIR-AF is an important method of examination for specifically visualizing melanin in the choroidal nodules of NF1 patients.

The irregular hyper-reflective foci in the choroid were seen using OCT in the same region that was found to be bright and patchy with NIR-R in cases 1 and 3, as reported by others. ${ }^{3,12}$ However, we also showed hyporeflective foci in the choroid using OCT in the same region that was found to be bright and patchy with NIR-R in case 2, who was 46 years old. Because the locations of the hypofluorescent regions revealed by ICGA corresponded to those of the bright patchy regions revealed by NIR-R and NIR-AF, and because regions of hyperfluorescence due to RPE alterations by FA were observed only in case 2, we speculated that long-standing choroidal nodules might induce focal choriocapillary atrophy, resulting in hypofluorescence on ICGA and hyporeflectivity of the choroid on OCT. On the other hand, the outer retinal structure of the outer limiting membrane and inner segment/ outer segment line did not show any abnormality on OCT. These results suggested that choroidal abnormalities in NF1 were localized in the choroid and did not affect the retina. However, more detailed histological studies are needed to clarify the effects on the retina.

In this study we showed that NIR-AF visualized dense melanin in the choroidal nodules, which appeared as bright patchy regions when visualized by NIR-R in three NF1 patients, and that the choroidal nodules detected by NIR-R showed irregular hyper-reflective or hyporeflective foci in the choroid on OCT without any effects on the retinal structure. NIR-AF should be employed together with NIR-R to detect melanin in the choroidal nodules of NF1 patients.

\section{Disclosure}

The authors report no conflicts of interest in this work.

\section{References}

1. Huson S, Jones D, Beck L. Ophthalmic manifestations of neurofibromatosis. Br J Ophthalmol. 1987;71:235-238.

2. Yasunari T, Shiraki K, Hattori H, Miki T. Frequency of choroidal abnormalities in neurofibromatosis type 1. Lancet. 2000;356:988-992.

3. Viola F, Villani E, Natacci F, Selcorni A, Melloni G, et al. Choroidal abnormalities detected by near-infrared reflectance imaging as a new diagnostic criterion for neurofibromatosis 1. Ophthalmology. 2012;119: 369-375.

4. Nakakura S, Shiraki K, Yasunari T, Hayashi Y, Ataka S, Kohno T. Quantification and anatomic distribution of choroidal abnormalities in patients with type 1 neurofibromatosis. Graefe's Arch Clin Exp Ophthalmol. 2005;243:980-984.

5. Gibbs D, Cideciyan AV, Jacobson SG, Williams DS. Retinal pigment epithelium defects in humans and mice with mutations in MYO7A: imaging melanosome-specific autofluorescence. Invest Ophthalmol Vis Sci. 2009;50:4386-4393.

6. Weinberger AWA, Lappas A, Kirschkamp T, et al. Fundus near infrared fluorescence correlates with fundus near infrared reflectance. Invest Ophthalmol Vis Sci. 2006;47:3098-3108.

7. Kellner U, Kellner S, Weinitz S. Fundus autofluorescence (488 nm) and near-infrared autofluorescence $(787 \mathrm{~nm})$ visualize different retinal pigment epitjelium alterations in patients with age-related macular degeneration. Retina. 2010;30:6-15.

8. Wolter JR. Nerve fibrils in ovoid bodies with neurofibromatosis if the choroid. Arch Ophthalmol. 1965;73:696-699.

9. Wolter JR, Gonzales-Sirit R, Mankin WJ. Neurofibromatosis of the choroid. Am J Ophthalmol. 1962;54:217-225.

10. Rajadhyaksha M, Grossman M, Esterowitz D, Webb RH, Anderson RR. In vivo confocal scanning laser microscopy of human skin: melanin provides strong contrast. J Invest Dermatol. 1995;104:946-952.

11. Keilhauer CN, Delori FC. Near-Infrared autofluorescence imaging of the fundus: visualization of ocular melanin. Invest Ophthalmol Vis Sci. 2006; 47:3556-3564.

12. Ayata A, Unal M, Ersanli D, Tatlipinar S. Near infrared fluorescence and OCT features of choroidal abnormalities in type 1 neurofibromatosis. Clin Experiment Ophthalmol. 2008;36:390-392.
Clinical Ophthalmology

\section{Publish your work in this journal}

Clinical Ophthalmology is an international, peer-reviewed journal covering all subspecialties within ophthalmology. Key topics include: Optometry; Visual science; Pharmacology and drug therapy in eye diseases; Basic Sciences; Primary and Secondary eye care; Patient Safety and Quality of Care Improvements. This journal is indexed on Submit your manuscript here: http://www.dovepress.com/clinical-ophthalmology-journal

\section{Dovepress}

PubMed Central and CAS, and is the official journal of The Society of Clinical Ophthalmology (SCO). The manuscript management system is completely online and includes a very quick and fair peer-review system, which is all easy to use. Visit http://www.dovepress.com/ testimonials.php to read real quotes from published authors. 